\title{
Professionalism: recognising and challenging unprofessional behaviour in the workplace
}

\author{
Authors: Jude Tweedie, ${ }^{A}$ Jan Cooper ${ }^{B}$ and Joydip Banerjee ${ }^{C}$
}

\section{Aims}

The aim of this study was to promote professionalism in practice by raising awareness of the prevalence and impact of unprofessional behaviours in healthcare.

\section{Methods}

A unique series of four interactive workshops were developed and delivered across the UK, enabling participants to recognise and manage unprofessional behaviour in the workplace. The workshop aim was to develop skills to identify and challenge unprofessional behaviour in the workplace, with the following learning objectives:

> participants will be able to describe unprofessional behaviours in the workplace

$>$ participants will be able to explain how this behaviour contributes to errors and safety incidents and fosters an unsafe environment

> participants will describe practical, evidence-based methods for managing unprofessional behaviours in the workplace.

The workshops were 3.5 hours in duration and combined interactive / small group work with a facilitated discussion, a short presentation of relevant literature and were split into three sections:

> Section 1 - participants sketch and discuss perceptions of unprofessional behaviours

> Section 2 - participants identify unprofessional behaviour within case studies created by the project team and consider the potential impact on patient safety

> Section 3-participants role-play challenging unprofessional behaviour utilising tools and models provided.

\section{Results}

Both quantitative and qualitative data were collected via written evaluation forms at each workshop. 69 (96\%) participants completed evaluation forms. Consultant clinicians constituted the largest group in all locations, accounting for $64 \%$ of total

Authors: ${ }^{A}$ Royal College of Physicians / University College London, London, UK; ${ }^{\mathrm{B}}$ General Medical Council, London, UK; ' University Hospitals of Leicester, Leicester, UK attendees.

$99 \%$ of participants reported that they would recommend the workshop to a colleague. In terms of content and delivery, $99 \%$ of participants rated the content as either good or very good and $96 \%$ rated the delivery as good or very good.

$97 \%$ of participants indicated that the workshop had led them to reflect on their practice and $90 \%$ reported that they would change their practice as a result of the workshop.

Thematic analysis of the qualitative data demonstrated a change in participants' perspectives upon completing the workshop.

Participants described:

> more confidence in recognising and challenging unprofessional behaviour

> being more proactive in managing disruptive behaviour earlier

> clear appreciation for the evidence base.

\section{Conclusions}

The evaluative data suggest that the teaching methods and approaches used were successful in the achievement of both the project and workshop objectives. Of particular note are the data in which participants state they would change their practice as a result of workshop attendance and had increased confidence in tackling unprofessional behaviour in their workplaces. The general comments also indicate that there is a need and desire for further workshops. The authors are currently developing a workshop to be rolled out nationally based on the project outcomes outlined above.

\section{Conflict of interest statement}

This specific work was funded by the General Medical Council. The overall project has funding from the Royal College of Physicians charitable funds, University of Oxford and the Nuffield Foundation. 